\title{
Changing memory of food enjoyment to increase food liking, choice and intake
}

\author{
Eric Robinson*, Jackie Blissett and Suzanne Higgs \\ School of Psychology, University of Birmingham, Edgbaston, Birmingham B15 2TT, UK \\ (Submitted 4 May 2011 - Final revision received 9 November 2011 - Accepted 27 November 2011 - First published online 3 January 2012)
}

\section{Abstract}

Novel ways to increase liking and intake of food are needed to encourage acceptance of healthier food. How enjoyable we remember food to have been is likely to be a significant predictor of food choice. Two studies examined whether remembered enjoyment of eating a food can be increased and whether this makes individuals more likely to eat that food in the future. In Study One, a simple manipulation of instructing participants to rehearse what they found enjoyable about a food immediately after eating it was used to increase remembered enjoyment (relative to controls). In a separate study; Study Two, the effect of increasing remembered enjoyment on food choice was tested by examining whether the manipulation to increase remembered enjoyment resulted in participants choosing to eat more of a food as part of a later buffet lunch. The experimental manipulation increased remembered enjoyment for the food (Study One). A change in remembered enjoyment was shown to have a significant effect on the amount of a food participants chose to eat the following day for lunch (Study Two). The present studies suggest that remembered enjoyment can be increased via a simple act of rehearsal, resulting in a later increase in the amount of food chosen and eaten. Interventions based on altering remembered enjoyment of healthy food choices warrant further investigation.

\section{Key words: Memory: Food choice: Food liking}

Dietary choices have significant impact on health and wellbeing $^{(1-3)}$. One factor that is thought to influence eating behaviour is food liking. Although recent research has suggested that liking may be less important for some eating behaviours such as portion size selections ${ }^{(4)}$, studies have consistently found that with regards to making choices between foods, evaluations of how much one likes a food is important ${ }^{(5,6)}$. Thus, novel ways to increase enjoyment and liking of healthier foods may be useful in shaping food choice.

The roles that memory and learning play in the acquisition of food likes and dislikes have been appreciated for some time ${ }^{(7)}$. When making food choices, we are reliant on our memory for how much we enjoyed eating that food previously because many food choices will be made in the absence of direct sensory contact with a food ${ }^{(8,9)}$. Hence, remembered enjoyment is likely to play a significant role in shaping food choices. In line with this premise, when individuals consider whether a repeat experience will be enjoyable, memories of similar past experiences shape these predictions $^{(8)}$. Similarly, research from cognitive psychology supports this by showing that a manipulation to produce a less negative memory for a past medical experience resulted in an increased likelihood of returning for repeat surgery ${ }^{(10)}$.
Some research has implicated memory for recent eating experiences in the regulation of food intake. For example, Higgs $^{(11,12)}$ has shown that enhancing memory for a recent eating experience can result in reduced intake later in the day, presumably because memory for ingested food informs decisions about how much food is appropriate to be eaten. In addition, neuropsychological studies show that damage to areas of the brain associated with memory can result in patients becoming poor at food intake regulation ${ }^{(13,14)}$. Yet, up till now, little research has examined how remembered enjoyment makes an impact on food choice. Given that there is also evidence that memory for past experience can be manipulated and changed ${ }^{(15,16)}$, this suggests a potential way to increase food liking and intake.

The aim of the two experiments presented here was to examine if a simple intervention could be used to increase remembered enjoyment of a food (Study One) and whether this would result in individuals choosing to eat more of the food at a later date (Study Two). In Study One, participants ate a lowenergy ready meal. To change remembered enjoyment, we used a simple rehearsal strategy. Rehearsing information changes the way it is encoded in memory, making it more memorable ${ }^{(17)}$. Thus, participants in an experimental condition

Abbreviation: MANCOVA, multivariate ANCOVA

*Corresponding author: E. Robinson, fax +44 121 4144987, email e.robinson@bham.ac.uk 
thought about what they found enjoyable about the meal immediately after consumption, in order to make the enjoyable aspects of the meal more salient in memory. A day later, participants returned and rated remembered enjoyment of the food. In a separate study (Study Two), we examined whether the manipulation used to increase remembered enjoyment would also result in participants choosing and eating more of a target food as part of a lunchtime buffet the following day.

We hypothesised that in Study One, the rehearsal manipulation would change memory for the meal, resulting in an increase in how enjoyable participants remembered the meal to be, compared with the control group (Study One). We also hypothesised that in Study Two, the manipulation to increase remembered enjoyment would result in participants choosing more of a target food for lunch the following day (Study Two).

\section{Study One \\ Participants}

A total of fifty-eight psychology undergraduates (forty-eight females and ten males; age 20.2 (SD 3.2) years; BMI 23.3 (SD 4.4$) \mathrm{kg} / \mathrm{m}^{2}$ ) participated in exchange for course credit. Participants were undergraduate students recruited from the School of Psychology research participation scheme at the University of Birmingham. Advertisement was through an online portal in which participants signed up to time slots in advance of study participation. To disguise the aims of the study, it was advertised as a two-part study on 'Social emotions, mood and food'. On arrival for their sessions, all participants provided signed consent for participation in the research. This study was conducted according to the guidelines laid down in the Declaration of Helsinki and all procedures involving human subjects were approved by the University of Birmingham ethics committee. Written informed consent was obtained from all subjects.

\section{Overview}

Participants consumed a lunchtime meal and halfway through the meal, rated their enjoyment of the food (online enjoyment). On finishing the meal, the participants were assigned to an experimental or control group activity (memory manipulation); and $24 \mathrm{~h}$ later, the participants returned and rated how enjoyable they thought the meal was (remembered enjoyment).

\section{Experimental groups}

The participants were assigned to one of four conditions:

(1) Enjoyable aspects of meal rehearsal (experimental), n 14: After eating the meal, participants in this group were asked to 'write down your thoughts on the enjoyable aspects of the meal, providing as much detail as possible'. Thus, this group rehearsed the enjoyable aspects of the meal.

(2) Other recent experience rehearsal (control), n 15: Participants in this group were asked to 'write down your thoughts on the enjoyable aspects of your journey to campus today, providing as much detail as possible'. This group controlled for effects of rehearsing a recent experience.

(3) Other recent meal rehearsal (control), n 14: Participants in this group were asked to "write down your thoughts on the enjoyable aspects of a meal you ate yesterday, providing as much detail as possible'. This group controlled for any effect of thinking about enjoying recently eaten food.

(4) Neutral meal rehearsal (control), n 13: Participants in this group were asked to 'think back to when you were eating the meal and answer the following questions: (1) List the meal ingredients; (2) How long did it take to eat the meal?; (3) Which utensils did you eat with? This group controlled for rehearsing the meal without concentrating on its enjoyable aspects.

\section{Procedure}

The sessions took place on weekdays at lunchtime. Participants were informed that the study would involve eating a lunchtime meal and returning the following day at the same time. Participants were then seated alone in the laboratory. After answering questions on demographics, and rating baseline hunger ('how hungry are your right now?' $10 \mathrm{~cm}$ visual analogue scale, anchors; 'not at all' and 'extremely'), participants were provided with a mock personality questionnaire to corroborate the study's cover story. On completion, the lunchtime meal was served on a white plate (diameter $25 \mathrm{~cm}$ ). The meal was a Heinz Weight Watchers Tomato \& Basil Chicken ready meal (249 kcal (1042 kJ)). The meal consisted of chicken fillets in a sauce with tomato, courgette, pepper, basil and potato wedges. Participants were provided with a knife and fork to eat the meal.

After $2 \mathrm{~min}$, the experimenter returned and asked the participants to stop eating and complete the online meal questionnaire. This questionnaire included a measure of meal enjoyment (online enjoyment): participants rated the extent to which they agreed with the statement: 'the meal is pleasant' (5-point Likert scale, strongly disagree-strongly agree). To disguise the aims of the study, participants also rated how 'relaxed', 'sad', 'awake', 'nervous' and 'stressed' they were, using the same rating scales. Participants informed the experimenter when they had completed the questionnaire and were left alone to finish the meal. On completion, the experimenter returned and took away the plate and utensils. Participants then completed the experimental manipulation.

Following this, $24 \mathrm{~h}$ later, participants returned for the second session. After rating hunger (same scale as used in session 1), participants were provided with another mood questionnaire to corroborate the cover story, followed by three questions to assess remembered enjoyment. Participants answered three questions using separate $10 \mathrm{~cm}$ line scales. (1) 'Compared to an average lunch, yesterday's lunch was'; anchors (from left to right) - 'not at all enjoyable' and 'extremely enjoyable'. (2) 'I would enjoy eating the meal again'; anchors - 'not at all likely' and 'extremely likely'. (3) 'I would recommend the meal to a friend'; anchors - 'not at all likely' and 'extremely likely'. Participants then completed the cognitive restraint scale of 
the Three-Factor Eating Questionnaire ${ }^{(18)}$ before weight and height were measured using electronic digital scales and a stadiometer to calculate BMI $\left(\mathrm{kg} / \mathrm{m}^{2}\right)$. Finally, participants were asked what they thought the aims of the study were and thanked for their time.

\section{Statistical analysis}

The three remembered enjoyment questions were highly correlated, and so were collapsed to form one measure of remembered enjoyment. A multivariate ANCOVA (MANCOVA) was used to examine between-group differences for online and remembered enjoyment. Participant hunger level, BMI and restraint were considered to be potentially confounding variables, and so were entered as covariates in the model, to control for any potential bias on outcome measures and produce a more accurate assessment of the effect the condition had on outcome measures. Pillai's trace was chosen as the test statistic for the MANCOVA. An effect of condition on remembered enjoyment was hypothesised. If an effect was observed, to adjust for $\alpha$ error when making pairwise comparisons, a Dunnett's $t$ test was used to test whether the experimental manipulation resulted in an increase in remembered enjoyment compared with the three control conditions.

\section{Results}

PASW 18@ (SPSS, Inc.) was used for all data analyses.

When the sessions ended, one participant came close to guessing the aims of the study and one participant had finished the meal within $2 \mathrm{~min}$ (and was therefore unable to complete the 'online' questionnaire), and were hence removed from the analyses. Removal of the participant who came close to guessing the study aims did not change the pattern of results.

Online and remembered enjoyment. MANCOVA indicated that using Pillai's trace, there was a significant effect of condition on the outcome variables $(V=0 \cdot 24, \quad F(6,96)=2 \cdot 2$, $P<0.05)$. None of the covariates was significant in the model $(P$ values $>0 \cdot 10)$. As hypothesised, univariate analysis indicated no effect of group on online enjoyment $(F(3,52)=0 \cdot 19, P=0 \cdot 91)$ and a significant effect of group on remembered enjoyment $(F(3,52)=2 \cdot 90, P<0 \cdot 05)$. Dunnett's $t$ tests indicated that the experimental group had a significantly higher remembered enjoyment of the meal compared to all three control groups; other recent experience rehearsal group (mean difference $=1.9, P<0.05$ ), other meal rehearsal group (mean difference $=1.7, P<0.05$ ) and neutral meal rehearsal group (mean difference $=1 \cdot 6, P<0 \cdot 05$; see Table 1 ).

\section{Study Two}

\section{Participants}

A total of thirty-seven psychology undergraduates (thirty-two females and five males; age 20.1 (SD 2.8) years; BMI 22.6 $\left(\mathrm{SD} 3.9 \mathrm{~kg} / \mathrm{m}^{2}\right)$ ) participated in exchange for course credit. The experiment was advertised as a two-part study on 'Social emotions, mood and food'. Participation in Study One was defined as an exclusion criterion for participation
Table 1. Study One - online and remembered enjoyment of lunch meal (Mean values and standard deviations)

\begin{tabular}{|c|c|c|c|c|}
\hline & \multicolumn{2}{|c|}{$\begin{array}{c}\text { Online enjoy- } \\
\text { ment ( } 1-5 \\
\text { Likert scale) }\end{array}$} & \multicolumn{2}{|c|}{$\begin{array}{c}\text { Remembered } \\
\text { enjoyment } \\
(0-10 \mathrm{~cm} \text { line } \\
\text { scale })\end{array}$} \\
\hline & Mean & SD & Mean & SD \\
\hline Enjoyable aspects of meal ( $n 14)$ & $4 \cdot 2$ & 0.6 & $7 \cdot 0$ & $1 \cdot 2$ \\
\hline Other recent experience $(n 15)$ & $4 \cdot 0$ & 0.9 & $5 \cdot 2$ & $2 \cdot 2$ \\
\hline Other meal rehearsal $(n 15)$ & $4 \cdot 1$ & 0.8 & $5 \cdot 3$ & $2 \cdot 3$ \\
\hline Neutral meal rehearsal $(n 13)$ & $4 \cdot 1$ & 0.8 & $5 \cdot 4$ & $1 \cdot 8$ \\
\hline
\end{tabular}

in Study Two. This study was conducted according to the guidelines laid down in the Declaration of Helsinki and all procedures involving human subjects were approved by the University of Birmingham ethics committee. Written informed consent was obtained from all subjects.

\section{Overview}

Participants consumed samples of three foods, including a portion of Mediterranean vegetable quiche and rated their enjoyment of these foods in session 1 (online enjoyment). Hidden within another questionnaire, participants then completed the experimental or control group activity, specifically for the vegetable quiche (memory manipulation). Following this, $24 \mathrm{~h}$ later, the participants returned and, under the pretence of a different study, selected and ate a lunch from a buffet, which included the vegetable quiche (food choice and intake). Shortly afterwards, participants were instructed to think back to eating the foods in the first session and rate how enjoyable they remembered it to be. This final part of the study was a manipulation check for remembered enjoyment.

\section{Experimental groups}

The participants were assigned to one of two conditions:

(1) Enjoyable aspects of meal rehearsal (experimental), n 17: For the final question in the mock feedback questionnaire, participants in this group were asked to 'Please write down your thoughts on what was enjoyable about the Mediterranean Quiche?' Thus, this group rehearsed the enjoyable aspects of the meal.

(2) Neutral meal rebearsal (control), n 17: For the final question in the mock feedback questionnaire, participants in this group were asked to 'Think back to eating the Mediterranean Quiche. Please write down the ingredients in it and how long it took you to eat it?' This group controlled for rehearsing the meal without concentrating on its enjoyable aspects.

\section{Procedure}

The sessions took place on weekdays at lunchtime. On arrival, participants were informed by the experimenter that they 
were taking part in a study to examine how food intake and mood interact and that the first session was to check that participants were suitable to take part. They were informed that the second session would involve eating a lunchtime meal the following day. Participants were then seated alone in the laboratory. After answering questions on demographics and rating baseline hunger (see Study One), participants were provided with a mock suitability questionnaire, which included personality questions, in order to corroborate the cover story. On completion, the experimenter returned with three test foods. Participants were given small amounts to eat of each food; cocktail sausage $\times 1(7 \mathrm{~g})$, cheese and onion pastry $\times 1 \quad(11 \mathrm{~g})$ and Mediterranean roasted vegetable quiche $\times 1$ portion $(25 \mathrm{~g}$ ). All foods were purchased from Sainsbury's Supermarket, UK. The experimenter then explained to participants that they were required to eat and rate each of the foods, because they might be used the following day. Ratings of 'online enjoyment' were made on separate $10 \mathrm{~cm}$ visual analogue scales for each food (e.g. How enjoyable was the sausage?); anchors (from left to right) - 'not at all enjoyable' and 'extremely enjoyable.'

Shortly afterwards, the experimenter returned and provided participants with a 'feedback questionnaire'. The questionnaire consisted of two questions concerning how familiar the participants were with the foods and the final question was the hidden experimental manipulation. Experimental participants wrote down what they found enjoyable about the quiche and control participants were instructed to think back to eating the quiche and list the ingredients and how long it took to eat. Participants were left for $3 \mathrm{~min}$ to complete the measure. On completion, the experimenter informed participants that they were invited to return the following day at the same time. Participants arrived for session 2 of this study, $24 \mathrm{~h}$ later, and were seated alone in the laboratory and to further corroborate the study cover, completed a mock eighteen-item self-esteem scale and rated how awake, nervous, hungry, excited and stressed they felt by circling 'not at all', 'quite' or 'very' for each item.

Participants were then informed that they needed to eat lunch before completing further mood ratings. Participants were taken to a kitchen area, where the buffet was located. See the following sub-section for details on the buffet. After being informed to choose whatever they wanted and provided with a plate, the experimenter informed participants that they should take the food back to the laboratory to eat. To corroborate the cover story, participants were then informed that a second set of mood ratings would be left in the laboratory and that it was important that they completed them as soon as they finished eating. The experimenter was not present during food selection or eating. After eating their chosen lunch from the buffet and completing the mood ratings, participants were provided with the cognitive restraint scale of the Three-Factor Eating Questionnaire ${ }^{(18)}$, before being asked to guess the aims of the study. Manipulation check questions were then administered: participants were asked to think back to the foods eaten in the first session on the previous day and rate how enjoyable they were, using the same scales as in session 1 (measure of 'remembered enjoyment'). Weight and height were measured using electronic digital scales and a stadiometer, to calculate BMI $\left(\mathrm{kg} / \mathrm{m}^{2}\right)$.
The experimenter then calculated the amount of each food item selected from the buffet and noted down if any food was not consumed.

\section{Buffet foods}

In session 2, all participants were asked to choose some lunch from a buffet. The buffet foods were served on individual plates and in order to make participants believe that we were not monitoring food choice, plates were made to appear as though earlier participants had chosen foods from them also (all plates were approximately half-full). There were six foods in the buffet; $6 \mathrm{x}$ mini cheese and onion pastries $(69 \cdot 1 \mathrm{~g}, 343 \mathrm{kcal} / 100 \mathrm{~g}(1435 \mathrm{~kJ} / 100 \mathrm{~g})), 16 \times$ mini sausages $(97 \cdot 8 \mathrm{~g}, 295 \mathrm{kcal} / 100 \mathrm{~g}(1234 \mathrm{~kJ} / 100 \mathrm{~g})), 6 \times$ slices of Mediterranean roasted vegetable quiche $(150 \mathrm{~g}, 222 \mathrm{kcal} /$ $100 \mathrm{~g}(929 \mathrm{~kJ} / 100 \mathrm{~g})), 4 \times$ roast chicken and stuffing sandwich quarters $(78.8 \mathrm{~g}, 221 \mathrm{kcal} / 100 \mathrm{~g}(925 \mathrm{~kJ} / 100 \mathrm{~g})), 16 \times$ carrot sticks $(79.3 \mathrm{~g}, 26 \mathrm{kcal} / 100 \mathrm{~g}(109 \mathrm{~kJ} / 100 \mathrm{~g}))$ and $14 \times$ tortilla chips (25.8 g, $495 \mathrm{kcal} / 100 \mathrm{~g}(2071 \mathrm{~kJ} / 100 \mathrm{~g}))$.

\section{Statistical analysis}

As in Study One, MANCOVA was used to compare conditions, with participant hunger level, BMI and restraint accounted for as covariates. In line with the findings of Study One, we hypothesised that the experimental condition and control condition would not differ for online enjoyment of the target food, but would differ on remembered enjoyment. Therefore, a first MANCOVA served as a manipulation check, whereby online enjoyment and remembered enjoyment of quiche were entered as dependent variables. We hypothesised that the two conditions would differ in the total number of grams of target food chosen (quiche) and proportion of target $v$. non-target food chosen in lunch, i.e. in comparison to the control group, the experimental group would select more quiche and the proportion of their meal made up by quiche would be greater. Hence, in the second MANCOVA, total grams of quiche chosen and proportion of meal derived from quiche (in $\mathrm{g}$ ) served as dependent variables.

\section{Results}

PASW 18C (SPSS, Inc.) was used for all data analyses.

No participant came close to guessing the aims of the study; however, three participants included rehearsal of negative aspects of the quiche in the experimental writing condition, and so were removed from the analyses.

Online enjoyment and remembered enjoyment. MANCOVA indicated that using Pillai's trace, there was a significant effect of condition on the outcome variables $(V=0 \cdot 23$, $F(2,28)=4 \cdot 1, \quad P<0 \cdot 05)$. None of the covariates was significant in the model ( $P$ values $>0 \cdot 10$ ). As hypothesised, univariate analysis indicated no effect of group on online enjoyment $(F(1,29)=1 \cdot 0, P=0 \cdot 32)$ and a significant effect of group on remembered enjoyment, with higher remembered enjoyment observed in the experimental condition compared 
to the control condition $(F(1,29)=5 \cdot 6, P<0 \cdot 05)$. See Table 2 for online and remembered enjoyment values of food items.

Food choice and intake. All participants consumed the lunch items they selected; so choice and intake data are identical.

MANCOVA indicated that using Pillai's trace, there was a significant effect of condition on the outcome variables $(V=0 \cdot 23, F(2,28)=4 \cdot 2, P<0 \cdot 05)$. None of the covariates was significant in the model ( $P$ values $>0 \cdot 10)$. As hypothesised, univariate analysis indicated that total grams of quiche chosen differed by condition $(F(1,29)=6 \cdot 4$, $P=<0.05)$ and proportion of lunch derived from quiche differed by condition $(F(1,29)=8 \cdot 5, P<0 \cdot 05)$. In the experimental condition, $30 \%$ (SD 14.9) of total lunch was derived from quiche and in the control condition, 15.0\% (SD 16.5) of total lunch was derived from quiche. See Table 3 for amount of each food selected in grams.

To further examine food selection, we used a $2 \times 2 \chi^{2}$ to examine whether the experimental manipulation resulted in a greater likelihood of choosing quiche. In the experimental condition, fifteen out of seventeen participants chose quiche as part of their lunch, compared to eleven out of seventeen participants choosing quiche in the control condition. This difference was significant $\left(\chi^{2}(1)=4 \cdot 1, P<0 \cdot 05\right)$. Frequency of choice of the two foods that were consumed in session 1 , but were not subject to the memory manipulation (cocktail sausage and pastries), was also examined using $\chi^{2}$. Analyses indicated no significant differences for either food ( $P$ values $>0 \cdot 4)$.

\section{Discussion}

After thinking about the enjoyable aspects of a meal shortly after consumption, experimental participants in Study One remembered the meal to be significantly more enjoyable than controls when asked $24 \mathrm{~h}$ later. In Study Two, the same manipulation again resulted in a change to remembered enjoyment and a marked increase in choice and intake of a vegetable quiche selected as part of a lunch from a naturalistic buffet setting. The observed effect on food choice and intake is particularly striking. In Study Two, the experimental manipulation resulted in participants consuming close to

Table 2. Study Two - online and remembered enjoyment of foods* (Mean values and standard deviations)

\begin{tabular}{llllll}
\hline & $\begin{array}{c}\text { Experimental } \\
\text { condition } \\
(n \text { 17) }\end{array}$ & & & \multicolumn{2}{c}{$\begin{array}{c}\text { Control } \\
\text { condition } \\
(n 17)\end{array}$} \\
\cline { 2 - 3 } & Mean & SD & & Mean & SD \\
\hline Quiche online enjoyment & 5.2 & 2.6 & & 4.5 & 2.6 \\
Sausage online enjoyment & 6.4 & 1.9 & & 5.8 & 2.5 \\
Pastry online enjoyment & 5.1 & 2.7 & & 5.6 & 1.3 \\
Quiche remembered enjoyment & 5.8 & 2.7 & & 3.9 & 2.6 \\
Sausage remembered enjoyment & 6.3 & 1.5 & & 5.9 & 2.5 \\
Pastry remembered enjoyment & 5.2 & 2.9 & & 5.5 & 1.8 \\
\hline
\end{tabular}

* Mean enjoyment ratings, $0-10 \mathrm{~cm}$ scale, anchors; 'not at all enjoyable' and 'extremely enjoyable'.
Table 3. Study Two - amount chosen and consumed of each food (g) (Mean values and standard deviations)

\begin{tabular}{|c|c|c|c|c|}
\hline & \multicolumn{2}{|c|}{$\begin{array}{c}\text { Experimental } \\
\text { condition }(n 17)\end{array}$} & \multicolumn{2}{|c|}{$\begin{array}{c}\text { Control } \\
\text { condition }(n 17)\end{array}$} \\
\hline & Mean & SD & Mean & SD \\
\hline Quiche & $37 \cdot 7$ & 21.9 & $19 \cdot 7$ & 23.6 \\
\hline Pastry & $16 \cdot 8$ & $15 \cdot 1$ & $11 \cdot 0$ & 9.5 \\
\hline Sausage & $19 \cdot 2$ & $10 \cdot 9$ & $16 \cdot 2$ & $10 \cdot 7$ \\
\hline Sandwich & $24 \cdot 3$ & $16 \cdot 4$ & $28 \cdot 9$ & $15 \cdot 8$ \\
\hline Tortilla chips & $5 \cdot 3$ & $5 \cdot 2$ & $6 \cdot 0$ & 3.8 \\
\hline Carrot sticks & $14 \cdot 3$ & 6.5 & $18 \cdot 9$ & $12 \cdot 4$ \\
\hline
\end{tabular}

twice the amount of the quiche as the control group. Similarly, the difference in remembered enjoyment between the experimental and control conditions in Study One was also large. These findings are the first to suggest that simple manipulations to alter remembered enjoyment of food may have significant effects on liking and intake of food.

The use of stringent controls in Study One indicates that the observed effects are unlikely to be due to demand characteristics. In Study Two, the memory manipulation of rehearsing the enjoyable aspects of the vegetable quiche did not result in a change to remembered enjoyment or choice of any of the other food items, which suggests that the effect is specific to the rehearsed food. The detailed cover stories used across both studies also resulted in the aims of the research being well hidden.

We suggest that the manipulation altered remembered enjoyment through rehearsal changing the consolidation of the memory of the meal, which had later consequences on choice from the buffet. Alternatively, it is also possible that rehearsal of the enjoyable parts of a food encouraged the participants to believe that they enjoy eating the food, so they acted accordingly to maintain a consistent self-perception when choosing from the buffet ${ }^{(19)}$. Regardless of the exact mechanism, the effect on food choice in Study Two and changes to remembered enjoyment across both studies suggests that altering memory is likely to have strong behavioural consequences.

These studies have a number of strengths including the use of multiple control groups to test for the influence of demand characteristics or expectations on the results, as well as a naturalistic setting in which the food choice and intake task occurred. But there are some limitations. The study population was university students, who were in the healthy-weight range, and who were tested in a laboratory setting. This may limit the generalisability of the findings. Further research examining the effects of altering remembered enjoyment in more naturalistic settings would be of interest.

The findings of the present studies suggest that simple manipulations to increase remembered enjoyment may be of practical use to increase food liking and intake, which is in keeping with recent ideas that episodic memory may be an important determinant of food choice ${ }^{(20)}$. Similarly, other research has also shown that reducing remembered pain during a medical procedure can have beneficial effects on the likelihood of returning for repeat surgery ${ }^{(10)}$. The results 
presented here are also in line with work that has shown enjoyment of food to be a strong and consistent predictor of food choice and intake ${ }^{(5)}$, although our emphasis on memory provides a different perspective to examine how food choices and intake can be influenced.

Although we show one simple manipulation here of postmeal rehearsal, we have also reported that the final and most enjoyable moments of a meal have a disproportionately strong impact on remembered enjoyment ${ }^{(21)}$. Thus, studies utilising these biases may also serve to increase remembered enjoyment and intake. These findings may also be of particular practical use in children, as early experiences with foods are thought to be particularly important in the acquisition of likes and dislikes ${ }^{(22,23)}$. In terms of direct applications, food items that are designed to promote greater remembered enjoyment (through a particularly pleasant end or peak, for example) may be a potential way to increase liking and acceptance. The post-event rehearsal strategy adopted in the present studies could also be a potential strategy of increasing food liking in children, in a similar vein to behavioural strategies (such as mere exposure, flavour-flavour conditioning and modelling) that attempt to increase intake of lesser-liked foods in the home or at school. The increase in the amount chosen and intake observed in the second study should be taken note of ( $90 \%$ increase compared to control), suggesting that altering remembered enjoyment may have impact outside of the laboratory. For example, a similar increase in vegetable intake would be of clinical relevance, as a significant proportion of the population do not adhere to intake guidelines $^{(24)}$

We hope to have drawn attention to an important yet under-investigated determinant of food choice in remembered enjoyment, which may prove to have useful application. Interventions aiming to increase remembered enjoyment of healthy food items are worthy of future research.

\section{Acknowledgements}

The present work was funded/supported by the University of Birmingham. The authors' contributions in connection with this work were as follows: E. R. was responsible for the study design, data collection, analysis and preparation of the paper. S. H. was responsible for the study design, analysis and preparation of the paper. J. B. was responsible for the study design and preparation of the paper. The authors declare that there are no conflicts of interest.

\section{References}

1. Van Itallie T (1979) Obesity: adverse effects on health and longevity. Am J Clin Nutr 32, 2723-2733.

2. Steinmetz KA \& Potter JD (1996) Vegetables, fruit, and cancer prevention: a review. J Am Diet Assoc 96, 1027-1039.

3. Bray GA (2003) Risks of obesity. Prim Care 30, 281-299.
4. Brunstrom JM \& Rogers PJ (2009) How many calories are on our plate? Expected fullness, not liking, determines meal-size selections. Obesity 17, 1884-1890.

5. De Graaf C, Kramer FM, Meiselman LL, et al. (2005) Food acceptability in field studies with US army men and women: relationships with food intake and food choice after repeated exposures. Appetite 44, 23-31.

6. Pollard J, Greenwood D, Kirk S, et al. (2001) Lifestyle factors affecting fruit and vegetable consumption in the UK Women's Cohort Study. Appetite 37, 71-79.

7. Rozin P \& Vollmecke TA (1986) Food likes and dislikes. Annu Rev Nutr 6, 433-456.

8. Wirtz D, Kruger J, Napa-Scollon C, et al. (2003) What to do on spring break? The role of predicted, on-line, and remembered experience in future choice. Psychol Sci 14, 520-524.

9. Gilbert DT \& Wilson TD (2007) Prospection: experiencing the future. Science 317, 1351-1354.

10. Redelmeir DA, Katz J \& Kahneman D (2003) Memories of colonoscopy: a randomized trial. Pain 104, 187-194.

11. Higgs S (2005) Memory and its role in appetite regulation. Physiol Behav 85, 67-72.

12. Higgs S (2008) Cognitive influences on food intake: the effects of manipulating memory for recent eating. Physiol Behav 94, 734-739.

13. Higgs S, Williamson AC, Rotshtein P, et al. (2008) Sensory specific satiety is intact in amnesics who eat multiple meals. Psychol Sci 19, 623-628.

14. Rozin P, Dow S, Moscovitch M, et al. (1998) What causes humans to begin and end a meal? A role for memory for what has been eaten, as evidenced by a study of multiple meal eating in amnesic patients. Psychol Sci 9, 392-396.

15. Loftus EF, Miller DG \& Burns HJ (1978) Semantic integration of verbal information into a visual memory. J Exp Psychol Hum Learn 4, 19-31.

16. Braun KA (1999) Post-experience advertising effects on consumer memory. J Consum Res 25, 319-334.

17. Spitzer HF (1939) Studies in retention. J Educ Psychol 30, 641-656.

18. Stunkard AJ \& Messick S (1958) The three factor eating questionnaire to measure dietary restraint, disinhibition and hunger. J Psychosom Res 29, 71-83.

19. Bem DJ (1972) Self-perception theory. In Advances in Experimental Social Psychology, vol. 6, pp. 1-62 [L Berkowitz, editor]. New York, NY: Academic Press.

20. Robinson E, Blissett J \& Higgs S (2011) Recall of vegetable eating increases predicted enjoyment and intake of vegetables in UK university students. J Am Diet Assoc 111, 1543-1548.

21. Robinson E, Blissett J \& Higgs S (2011) The final and most enjoyable moments of eating experience influence remembered enjoyment. Appetite 57, 207-212.

22. Skinner JD, Carruth BR, Wendy B, et al. (2002) Children's food preferences: a longitudinal analysis. J Am Diet Assoc 12, 1638-1647.

23. Rollins BY, Loken E \& Birch LL (2010) Stability and change in snack food likes and dislikes from 5 to 11 years. Appetite $\mathbf{5 5}$, $371-373$.

24. Craig R \& Hirani V (2010) Health Survey for england 2009, Volume 1: Health and lifestyles. http://www.ic.nhs.uk/ webfiles/publications/003_Health_Lifestyles/hse09report/ HSE_09_Volume1.pdf (accessed February 2011). 